\title{
Looking at education through the microscope
}

\section{Suhas Eswarappa Prameela $\odot$, Patricia M. McGuiggan, Amy Brusini, Trevor W. Glenn and Timothy P. Weihs}

\begin{abstract}
Advanced research microscopes in universities can be used to enhance the education of STEM students, as demonstrated by initiatives at Johns Hopkins University that give students the opportunity to get hands-on experience with sophisticated microscopes.
\end{abstract}

\begin{abstract}
Like many disciplines, STEM education at the university level has undergone tremendous change in the past few decades. Today's STEM students have increased access to a range of specialized courses, research experiences for undergraduates and competitions centred on science projects and inventions. In the classroom, STEM instructors are moving away from traditional lecture-based teaching and are incorporating more active learning strategies, such as inquiry-based, collaborative and case-based learning. Complementing this student-centred approach, STEM departments are organizing lab tours, giving undergraduate research assistant positions and allowing access to research equipment typically reserved for graduate students, postdocs and research professors. For example, microscopes can
\end{abstract} play a critical role in opening up scientifically compelling topics to students, as well as giving them valuable skills and hands-on experience.

Undergraduate students typically have access to simple light microscopes in their practical sessions; these microscopes are logistically easy and low cost and can be used by many students at once. More sophisticated research microscopes, such as confocal or fluorescence light microscopes and scanning or transmission electron microscopes (TEMs), are more difficult to make available to undergraduates. However, there is much to be gained from leveraging these tools to enhance the education of undergraduate students, as they enable them to gain a competitive edge to obtain internships, jobs and research assistant positions in labs, and to enter graduate programs. At Johns Hopkins University (JHU), several researchers and STEM educators are involved in programs to integrate advanced characterization methods into the teaching and training of students through specialized classes, university-sponsored grants, government-sponsored internships and apprenticeships, and other externally funded projects. Here, we highlight the lessons learned from these initiatives in the hope that faculty and administrators at other universities can create a similar infrastructure to support grants, classes and programs aimed at expanding education in the field of microscopy.

\section{Integration of microscopy tools}

At JHU, we offer several integrated laboratory and lecture courses, including short, one-credit (equivalent to $\sim 40$ hours per semester) classes to undergraduate students taught by $\mathrm{PhD}$ students and post-doctoral scholars through programs such as Hopkins Engineering Applications \& Research Tutorials (HEART) and Special Opportunities for Undergraduate Learning (SOUL). These classes expose students to cutting-edge research work on campus and draw students from many scientific disciplines, as well as from the social sciences and humanities. Classes range from gene therapy to machine learning.

Among such courses, Looking at atoms and viruses is a microscopy characterization class that focuses on teaching fundamentals of both light and electron microscopy. Every other class is spent in a laboratory probing different materials. Because students are not officially trained on the microscopes, they cannot independently operate the machines. However, students have the opportunity to handle samples and to make their own. Once a sample is ready, and the scope is tuned to the correct settings, students operate the position controls to navigate the sample space, change the magnification and make their own measurements, whereas the instructor or the lab manager carries out more sensitive operations such as debugging instrument errors. Two students take turns sitting beside the instructor during the demonstration. Given these logistics, the class enrolment is capped at between 10 and 12 students to ensure their learning experience is maximized.

In the advanced light microscopy lab, students look at blades of grass, fine grooves on keys or coins, human skin cells and mouse cells. Using a scanning electron microscope, students explore the microscale features of spiders, porous structures in butterfly wings and layers of paint from a wall. Using a TEM (FIG. 1a), students observe viruses, nanocrystals and atoms in metallic materials. The colourful contrasts from fluorescence and polarized light microscopy and the high level of magnification that can be achieved by electron microscopes are often intriguing for the students. Active learning exercises help students acquire core concepts behind the techniques more effectively than standard classroom instruction. For example, hands-on activities, such as building crystal structures using ball-and-stick models or virtual software tools, allow students to engage with the information in multiple ways.

In these classes, groups of two to three students also deepen their understanding of a microscopy technique by choosing a research paper from a preselected list and presenting the main findings to the rest of the class. The students' presentation topics range from studies of lithiation of silicon in batteries using operando TEM to imaging skin cells using fluorescence microscopy. Such focused activities help students appreciate the real-life applications of microscopes, as well as how research keeps pushing the capabilities of imaging methods.

One of the main lessons we have learned from the HEART and SOUL classes concerns the selection of samples to study. In the first iteration of these classes, we used only metallic samples, but many students in the biological sciences expressed interest in learning how microscopy could be applied to other materials. Since then, we have expanded the choice of samples by taking suggestions and feedback from the students.

At the School of Medicine, postgraduate students are offered characterization-oriented 

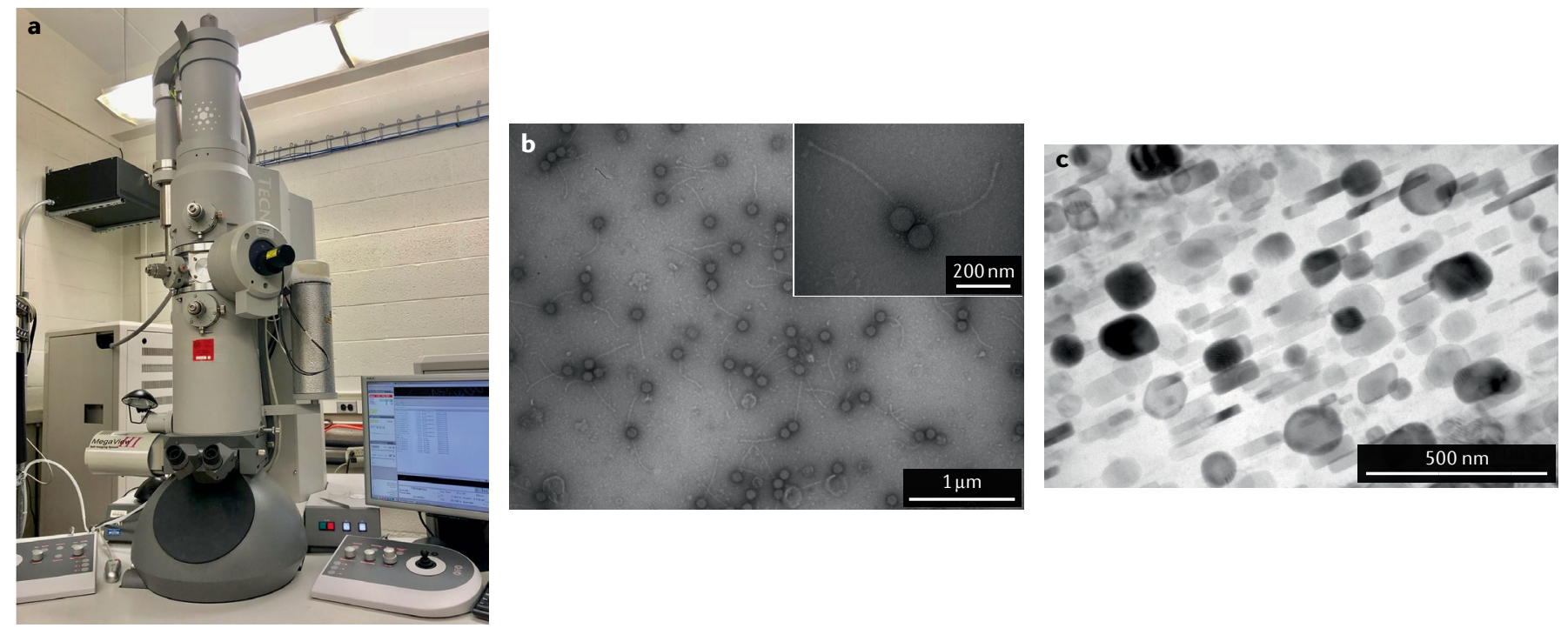

Fig. 1 | Teaching with microscopes. a |A transmission electron microscope (TEM) b |A TEM micrograph of bacteriophages from the Phage Hunters Project. c|A TEM image of nanocrystals in a magnesium alloy for the Materials in Extreme Dynamic Environments (MEDE) programme. Panel b courtesy of M. McCaffery and the Integrated Imaging Center, Johns Hopkins University.

classes similar to HEART and SOUL but focused on techniques used in medicine and the biological sciences. Students learn how confocal, fluorescence and electron microscopy techniques are used to study cells, evaluate disease processes and aid in diagnostic decision making. In virtual microscopy classes, students examine images using an online database, which allows them to see a wide range of images of pathological processes and contributes to a better understanding of how microscopy is used in medicine.

The use of microscopy in medicine is also taught at the undergraduate level. JHU, in partnership with Howard Hughes Medical Institute as part of the Science Education Alliance, launched the Phage Hunters Project (SEA-PHAGES program) a few years ago. In this program, two dozen freshman students dig up samples of soil and isolate a phage, a virus that infects a bacterium, and image it using a TEM (FIG. 1 b). Through this opportunity, students gain hands-on experience with microscopy and a greater understanding of the struggles and successes of sample preparation.

\section{Resources to aid microscopy education}

One way to strengthen education in microscopy is by collaborating with research faculty as well as professionals who manage microscopy facilities. These experienced professionals can provide pertinent information regarding the technical aspects of how microscopes function, which can be challenging to convey in a general classroom setting. For example, lab managers can show broken or replaced parts of the filament or apertures that go inside the microscope. By co-teaching small classes, they can provide support in designing activities and give exposure to the instruments and to their research activities in the short class periods.

These undergraduate microscopy initiatives have already started showing results at JHU. Of the students who took these classes, a few have joined research groups where they plan to use the microscopes and to gain more training. The students remarked that the HEART or SOUL classes prepared them to apply to study in the research laboratories of professors on campus. In addition, some senior undergraduate students have started using microscopes extensively before transitioning to Master's or PhD programs. A few of them mentioned that they knew they would have limited ability to take characterization-oriented classes during the first year of their new programs, thus having taken the HEART or SOUL classes before starting helped them to feel more prepared.

University-sponsored grants are important to improve courses. At JHU, the Center for Educational Resources provides technology fellowship grants to support a faculty member partnering with technology-savvy students to develop resources that enhance pedagogy and active learning in classes. For example, for the HEART and SOUL classes, technology fellowship grants were awarded to develop simulation tutorials for teaching microscopy both in class and in a virtual setting. Other universities are also building simulation tools for microscopy education. For example,
Microscopy Australia, in partnership with several universities, has developed an excellent open-source platform called Myscope Microscopy training. Through this website, anyone can access a wide variety of microscopy-related educational materials and operate virtual microscopes.

When developing and implementing specialized classes such as HEART or SOUL, soliciting feedback and making improvements is crucial to designing more effective class materials and syllabi. The addition of the simulation tutorials developed via the technology fellowship grants is one example. Initially, student evaluations averaged 3.8/5 for both the overall quality of the course and the effectiveness of instructor teaching. Once simulation tutorials, more lab visits and active learning exercises were incorporated, the ratings improved to $4.6 / 5$ for overall quality of the course and 4.3/5 for the effectiveness of instructor teaching.

Lastly, we are training several undergraduate students in advanced microscope techniques as part of their undergraduate research experience in laboratories and for senior design projects. For example, the US Army sponsors two programs, the Undergraduate Research Apprenticeship Program (URAP) and the Extreme Science Internships (ESI), through which students participate in an actual research project that has military applications. For example, students participated in the Materials in Extreme Dynamic Environments (MEDE) programme, which focuses on developing materials for lightweight armour. The nanocrystals analyzed by the students 
serve to strengthen the metallic materials being developed (FIG. 1c). These programs bring undergraduate students closer to the ongoing research on campus and result in students gaining internships or jobs in government or industry after graduation.

\section{Online teaching}

Universities across the world are transitioning to online or hybrid teaching owing to the COVID-19 pandemic. Also, as fewer students can be accommodated safely in teaching laboratories, this results in less exposure to experimental techniques. These drawbacks may be mitigated by developing educational tools, engaging students using virtual active learning exercises and giving simulation tutorials to bolster online learning experiences. At JHU, the next step is to procure mixed reality headsets through university-sponsored Digital Education \& Learning Technology Acceleration grants. We have procured HoloLens to enable students to virtually enter a microscope laboratory and operate the microscope remotely by communicating with an experienced user who physically controls the scopes. New tools, nurturing programs and focused grants can accelerate microscopy education on campuses, benefitting many students and enhancing their future careers.
Suhas Eswarappa Prameela (iD) $1,2 \otimes$, Patricia M. McGuiggan', Amy Brusini', Trevor W. Glenn ${ }^{4}$ and Timothy P. Weihs ${ }^{1,2}$

'Department of Materials Science and Engineering, Johns Hopkins University, Baltimore, MD, USA.

${ }^{2}$ Hopkins Extreme Materials Institute, Johns Hopkins University, Baltimore, MD, USA

${ }^{3}$ Center for Educational Resources, Johns Hopkins University, Baltimore, MD, USA

4Johns Hopkins School of Medicine, Baltimore, MD, USA.

凶e-mail: seswara3@jhu.edu

https://doi.org/10.1038/s41578-020-00246-z

Author contributions

S.E.P. wrote the majority of the manuscript and all authors contributed to the review and editing of the manuscript.

\section{Competing interests}

The authors declare no competing interests. 